Geopolítica(s) Revista de estudios sobre espacio y poder ISSN: 2172-3958

\title{
En memoria de Ron Johnston ${ }^{1}$
}

Ryan Weichelt ${ }^{2}$

Cómo citar: Weichelt, R. (2020). En memoria de Ron Johnston. Geopolitica(s). Revista de estudios sobre espacio y poder, 11(1), 25-27.

En 2003, en la reunión anual de la Asociación de Geógrafos Americanos (AAG) en Nueva Orleans (Louisiana) presenté uno de mis primeros trabajos como estudiante de maestría; en realidad, era un análisis bastante simplista de los resultados electorales en las comunidades universitarias (college communities) en Estados Unidos.

Cuando se publicaron los horarios de las sesiones, me quedé asombrado con la incorporación de Ron Johnston como comentarista en mi sesión sobre las nuevas tendencias de investigación en la geografía electoral estadounidense. Rápidamente me pregunté ¿cómo pude ser puesto en una sesión con "él”, con Ron Johnston? Rápidamente llegaron el miedo y la ansiedad de tener que presentar frente a él. Desgraciadamente, el avión de Ron se retrasó y no pudo asistir a la sesión, pero esta pequeña historia es un testimonio de lo que Ron Johnston significó no solo para la geografía electoral, sino también para la disciplina de la geografía.

En todo el caos actual del mundo, la pérdida se ha convertido en un lugar demasiado común. Para la escuela de Geografía, la punzada de la pérdida se sintió más con el repentino fallecimiento de Ron Johnston el 29 de mayo de 2020. En los ámbitos de la Geografía Humana y Política, pocos nombres se destacan más que Ron Johnston. Aunque mis intercambios personales con él fueron bastante limitados, mis interacciones intelectuales con su trabajo sí fueron frecuentes, ya que pocos académicos pueden compararse con la productividad de la investigación de Ron.

Como geógrafo electoral, la pérdida de Ron Johnston para este pequeño subconjunto de geografía política será dolorosa. Sin embargo, en su pérdida, la dedicación y la pasión de Ron por la disciplina ha proporcionado a los académicos actuales y futuros un itinerario a seguir. Hay un libro que debe leer cualquier geógrafo electoral principiante: Geography of Elections, de Peter Taylor y Ron Johnston (1979). Este libro mostró al público en general la forma en la que la geografía puede ayudar a comprender las elecciones y la política a nivel local. En particular, el capítulo cinco, "El efecto de vecindario", tuvo una profunda influencia en mí. Este capítulo

Traducción de María Lois.

Department of Geography and Anthropology, University of Wisconsin-Eau Claire (Estados Unidos)

E-mail: weicherd@uwec.edu 
analizaba “... las razones por las cuales diferentes personas en el mismo lugar votan de la misma manera, mientras que personas similares en diferentes lugares votan de diferentes maneras" (p.222).

La capacidad de combinar una multitud de teorías de ciencias sociales con una comprensión geográfica de la actividad humana fue lo que mejor hizo Ron. Su continuo trabajo con análisis de vecindarios también cimentó su contribución en términos de avances metodológicos que combinaban métodos cuantitativos y estudios electorales. Estos estudios no solo incluían análisis de las votaciones, sino también estudios sobre financiamiento de campañas, formación de límites y estudios urbanos - por nombrar algunos-. Su libro pionero, Multivariate Statistical Analysis (2008), reunió todas estas amplias experiencias que se convirtieron en una lectura común para muchos estudiantes de posgrado de todo el mundo.

A lo largo de una carrera académica que se extendió durante cinco décadas, Ron Johnston fue autor o coautor de 467 artículos de revista, 127 capítulos de libro, 18 libros y editor o coeditor de 10 libros más. Sin embargo, creo que su legado estará en su capacidad para empujar a otros geógrafos e investigadores a cuestionarse continuamente sus roles en su disciplina. Una de mis primeras lecturas de Ron Johnston fue su texto de 2001 'Out of the 'moribund backwater': territory and territoriality in political geography”, publicado en Political Geography. Este artículo condensaba la historia de treinta años de geografía política, pero también cuestionaba el lugar del estudio de la política por geógrafos. Si bien otorgaba un merecido crédito a los impactos de otros geógrafos políticos importantes como Peter Taylor, también planteaba desafíos a otros geógrafos políticos para definir su espacio en un mundo en constante cambio.

Estos desafíos no fueron sólo para la geografía política, sino a la comunidad académica en general. En 2011 fue autor de un artículo bastante destacado en The Professional Geographer, "Promoting Geography (or Part of It) - Yet Again", en el que criticaba magistralmente a los no geógrafos por su falta de comprensión del campo, y a los geógrafos por su falta de confianza en su propia disciplina. Johnston escribía:

Los geógrafos, tanto individual como colectivamente y mucho más que sus colegas en la mayoría de las otras ciencias de la tierra, ambientales y sociales, con frecuencia parecen algo paranoicos sobre su disciplina. Se sienten relativamente ignorados y subvalorados, ocupando una posición inferior en la división académica del trabajo; no se reconoce la calidad y relevancia de su investigación y, en consecuencia, no son demandados con tanta frecuencia como les gustaría para aplicar su experiencia en responder a los principales problemas sociales. (p.325).

Del mismo modo, el deseo de Johnston de mostrar una historia de la geografía también le llevó a promover una plataforma para proporcionar recursos para los geógrafos, como se ve en su volumen editado de 1981 en el Diccionario de Geografía Humana (en su quinta edición y con traducción al castellano), pero una vez más desafió a los geógrafos a no tener miedo de promover el pensamiento espacial en un artículo de 2018, "Quantitative Methods I: The world we lost - or where we started from", y en otro de 2019, “A Classic that wasn't: Statistical Geography and paths only later taken". 
La geografía está en un lugar mucho mejor gracias a Ron Johnston. Todos somos afortunados de que Ron haya dejado un tesoro tan grande de sus pensamien-

tos, investigaciones e ideas. Este pequeño homenaje sólo resalta algunas de sus muchas contribuciones al campo. ¡Pero creo que la mejor manera de honrar a Ron Johnston es leer algunas de sus muchas publicaciones, y tomar en serio sus desafíos para avanzar en la disciplina y no tener miedo a declararse geógrafo!

\section{Referencias}

Johnston, R. J. (1980). Multivariate Statistical Analysis in Geography: A Primer on the General Linear Model. London: Longman.

Johnston, R. J. (Ed.). (1981). The Dictionary of Human Geography. Oxford: Blackwell Reference. [Trad. al castellano de la $2^{\mathrm{a}}$ ed, Johnston, R. J., Gregory, D., y Smith, D. M. (Eds.). (1987). Diccionario de Geografia Humana. Madrid: Alianza Editorial].

Johnston, R. J. (2001). Out of the "moribund backwater": territory and territoriality in political geography. Political Geography, 20(6), 677-693.

Johnston, R. J. (2011). Promoting Geography (or Part of It)-Yet Again! The Professional Geographer, 63(3), 325-331.

Johnston, R. J., Harris, R., Jones, K., Manley, D., Wang, W. W., y Wolf, L. (2019). Quantitative Methods I: The World We Have Lost - or Where We Started From. Progress in Human Geography, 43(6), 1133-1142.

Johnston, R. J., y Jones, K.( 2020). A Classic That Wasn't: Statistical Geography and Paths Only Later Taken. Progress in Human Geography, 44(2), 357-373.

Taylor, P. J., y Johnston, R. J. (1979). Geography of Elections. New York: Routledge. 